\section{Short-term changes in the optic nerve head and visual field after trabeculectomy}

M Figus', S Lazzeri' ${ }^{1}$, M Nardi' ${ }^{1}$ MP Bartolomei', A Ferreras ${ }^{2}$ and $P$ Fogagnolo ${ }^{3}$

\section{Introduction}

Primary open-angle glaucoma (POAG) is a slowly progressive disease characterized by degeneration of retinal ganglion cells with loss of the retinal nerve fiber layer (RNFL) and thinning of the neuroretinal rim. Clinical and instrumental examination of RNFL and of the optic nerve head (ONH) is a key component in establishing the diagnosis and monitoring the course of the disease, as well as the functional evaluation by means of visual field (VF) testing.

A standard surgical approach for glaucoma is trabeculectomy, a filtering procedure which induces a sudden intraocular pressure (IOP) reduction and is effective in reducing glaucoma progression. ${ }^{1}$ Partial regression of glaucomatous damage to RNFL after IOP reduction following glaucoma surgery has been documented in congenital and infantile glaucoma. ${ }^{2,3}$ In adult POAG, some reports have shown a nonsignificant change in retinal thickness after IOP reduction. ${ }^{4,5}$ Reports about the ONH have demonstrated a significant change of some parameters and reversal of cupping. ${ }^{6-8}$

Structure and function relationship after trabeculectomy have just been previously studied. Some reports have suggested that improvement in disk appearance would correspond to improvement of visual function, ${ }^{9,10}$ other authors have not shown any significant change of visual field after trabeculectomy. ${ }^{11}$

Due to controversy on short-term results of functional and morphological data after trabeculectomy, we decided to conduct a shortterm prospective study to evaluate the changes in neural topography and function after glaucoma filtration surgery in adults with POAG, and investigated the relationship between IOP reduction and functional and anatomical changes.

\author{
${ }^{1}$ Department of \\ Neurosciences, \\ Ophthalmology, University \\ of Pisa, Pisa, Italy \\ ${ }^{2}$ Miguel Servet University \\ Hospital, Instituto Aragonés \\ de Ciencias de la Salud, \\ University of Zaragoza, \\ Zaragoza, Spain \\ ${ }^{3}$ G.B.Bietti Foundation- \\ IRCCS (Istituto di Ricovero \\ e Cura a Carattere \\ Scientifico), Rome, Italy
}

Correspondence: M Figus, Via B. Buozzi, 19, 55045 Pietrasanta (Lucca) Italy Tel: + 39058471117 ; Fax: + 39050992976. E-mail: figus@ocupisa.it

Received: 7 January 2011 Accepted in revised form: 5 April 2011

Published online: 27 May 2011 


\section{Materials and methods}

\section{Patients}

Subjects enrolled in the study were 67 consecutive POAG requiring trabeculectomy at Pisa University (Italy) from September 2007 to May 2008. All patients signed an informed consent, and the design of the study adhered to the tenets of the Declaration of Helsinki.

Inclusion criteria were perimetric POAG, pre-operative uncontrolled IOP (mean office-hour IOP $>20 \%$ compared with target pressure) despite maximumtolerated medical therapy, best visual acuity equal or above to $20 / 100$, IOP reduction after trabeculectomy higher than $25 \%$ compared with baseline, post-operative IOP higher than $5 \mathrm{~mm} \mathrm{Hg}$, and ability to return in the 6 months following surgery for scheduled visits.

Exclusion criteria were: allergy to medication used during and after surgery, previous intraocular surgery (except cataract surgery at least 180 days before inclusion), significant co-morbid disease that could interfere with the follow-up and reassessment of the bleb.

\section{Study procedures}

Visits were performed 4 weeks before surgery $( \pm 3$ weeks), and at 3 months ( \pm 2 weeks) and 6 months $( \pm 3$ weeks) after surgery, and included: full ophthalmological examination, IOP measurement with Goldmann applanation tonometry, visual field (VF) tests, and imaging of the $\mathrm{ONH}$ with the Heidelberg Retina Tomograph Version II (Heidelberg Engineering, Dossenheim, Germany).

The IOP value was the mean of three measurements performed in each eye at the same hour of the day (at $8.00 \mathrm{am}$, with an interval of $\pm 30 \mathrm{~min}$ ).

Standard Automated Perimetry (SAP) tests were performed using a Humphrey Field analyzer, model HFA II-750 (Zeiss Humphrey Systems, Dublin, CA, USA), with the Swedish Interactive Threshold Algorithm (SITA) Standard 30-2 strategy. All patients were experienced with SAP, and therefore it was not necessary to rule out learning effect. Abnormal SAP results were considered to be a reproducible glaucomatous VF loss in the absence of any other abnormalities to explain the defect. Mean VF defects were classified with Glaucoma Staging System (GSS) ${ }^{12}$ within stage 4 . The pre-operative and 3-months and 6-months post-operative mean deviation (MD) and pattern standard deviation (PSD) indexes were used for the analysis. During the study, the test was repeated if fixation losses were greater than $15 \%$ and false positive or false negative rates were greater than $20 \%$.

Analysis of the optic disk was performed using HRT II, which provides topographic measurements of the $\mathrm{ONH}$ with a $15^{\circ}$ field of view. The margin of the optic disks was manually traced by the same glaucoma specialist (MF), who was masked to the patients' identity and clinical history, defining the inner edge of the Elschnig's ring with at least a five-point contour line. In order to maintain a quality control over the images used in the analysis, mean topography images had to satisfy two criteria: mean topography standard deviation less than $40 \mu \mathrm{m}^{13}$ and a refractive error change (or change in focus setting) less than two diopters within the three retests. ${ }^{14}$ The following global stereometric parameters were evaluated: mean retinal nerve fiber layer thickness, rim area, rim volume, cup area, cup volume, cup/disk area ratio, maximum cup depth.

\section{Statistical issues}

Formal sample size was calculated in order to assess the change in RNFL (which was considered as the primary outcome of our study) between the pre- and postinterventional periods, if one existed. We assumed as clinically relevant the changes occurring between moderate and severe glaucoma cases, ${ }^{15}$ and we therefore estimated $\Delta$ (the difference between post- and preinterventional RNFL) at 20 microns and $\sigma$ at 40 microns (20). The following formula for continuous, paired data were used:

$$
n=\frac{\left[\Phi^{-1}(\alpha / 2)+\Phi^{-1}(\beta)\right]^{2} \sigma^{2}}{\Delta^{2}}
$$

Using $\alpha=0.05$ and $\beta=0.90$, this formula would enumerate 50 patients. In order to reduce the possibility of II-type error, we set $\beta$ at 0.95 , and we therefore calculated our sample size to 55 patients.

All statistical analyses were calculated using SPSS (version 15.0; SPSS Inc., Chicago, IL, USA) statistical software. First, the Kolmogorov-Smirnov test was applied to check that the data were normally distributed. The $t$-test for paired data was used to compare the variables before and after surgery. Linear regression analysis was used to determine the relationship between IOP reduction and VF changes, between IOP reduction and $\mathrm{ONH}$ modifications, and between VF changes and ONH modifications.

\section{Results}

In all, 67 patients were enrolled in the study, but eight patients were excluded because surgical IOP reduction was less than $25 \%$ and three patients were excluded because mean topography standard deviation was higher than $40 \mu \mathrm{m}$. Statistical analyses were therefore performed on 56 eyes. The clinical characteristics of the study 
population are described in Table 1 . None of the participants had any surgical complication or macular or disk edema. Sutures were released in 39 eyes $(69.6 \%)$ $13 \pm 3$ days after surgery; 17 patients $(30.4 \%)$ required needling of the bleb within $3 \pm 1$ weeks from surgery; 14 patients (25\%) needed topical therapy to achieve target IOP.

Pre-surgery visual acuity was 20/40 or better in 49 patients $(87.5 \%)$ and worse than $20 / 40$ in seven patients (12.5\%). Post-surgery visual acuity was $20 / 40$ or better in 53 patients $(94.6 \%)$ and worse than $20 / 40$ in three patients $(5.4 \%)$.

IOP decreased from $24.4 \pm 5.0 \mathrm{~mm} \mathrm{Hg}$ pre-operatively to $12.1 \pm 3.1 \mathrm{~mm} \mathrm{Hg}(P<0.001)$ and $10.6 \pm 2.8 \mathrm{~mm} \mathrm{Hg}$ $(P<0.001)$, respectively, at 3 and 6 months after trabeculectomy; this corresponded to a reduction of $48 \pm 18 \%$ at 3 months and of $54 \pm 20 \%$ at 6 months (Table 2).

The average pre-operative MD and PSD were $-14.05 \pm 3.37$ and $8.58 \pm 1.79 \mathrm{~dB}$, respectively, (Table 1). At 3 and 6 months, respectively, MD decreased to $-13.35 \pm 3.26$ and $-13.58 \pm 3.54 \mathrm{~dB}(P=0.06$ and

Table 1 Sample clinical characteristics

\begin{tabular}{lccr}
\hline & Minimum & Maximum & Mean $\pm S D$ \\
\hline Age (years) & 53 & 82 & $64.2 \pm 14.9$ \\
BCVA (Snellen) & $20 / 100$ & $20 / 20$ & $20 / 32 \pm 6.50$ \\
IOP (mm Hg) & 22 & 38 & $24.4 \pm 5.00$ \\
C/D & 0.79 & 0.53 & $0.62 \pm 0.25$ \\
Pachymetry (microns) & 482 & 563 & $534 \pm 17.8$ \\
MD SAP (dB) & -19.56 & -11.02 & $-14.05 \pm 3.37$ \\
PSD SAP (dB) & 7.23 & 12.15 & $8.58 \pm 1.79$ \\
\hline
\end{tabular}

Abbreviations: BCVA, best-corrected visual acuity; C/D, vertical cup/ disc ratio in stereophotographs; IOP, baseline intraocular pressure (without treatment); MD, mean deviation; PSD, pattern standard deviation; SD, Standard deviation; SAP, standard automated perimetry.
$P=0.06)$, and PSD increased to $9.20 \pm 1.86$ and $8.97 \pm 1.52 \mathrm{~dB}(P=0.08$ and $P=0.06)$ (Table 2$)$.

Mean RNFL thickness showed statistically significant changes (Figures 1a and b): the thickness increased by $0.05 \mathrm{~mm}$ out of $0.19 \pm 0.034 \mathrm{~mm}$ baseline at 3 months $(P=0.05)$, and $0.03 \mathrm{~mm}$ at 6 months $(P=0.05)$. The mean value of RNFL in normal elderly patients is $0.23 \pm 0.07 \mathrm{~mm},{ }^{16}$ even if in the printout of the HRT II the normal range of RNFL is $0.20-0.32 \mathrm{~mm}$. No statistically significant changes were found for rim area, rim volume, maximum cup depth, cup area, cup volume, and cup/ disk area ratio $(P>0.05)$ (Table 2$)$.

Changes in RNFL had a significant association with changes in IOP 3 months after surgery $(P=0.05)$; the correlation between the two variables was negligible $\left(r^{2}=0.01201\right)$. This association was statistically significant also at month $6(P=0.05)$.

Changes in rim parameters (area and volume) did not show statistically significant association with $\triangle \mathrm{IOP}$ from basal values (rim area, $P=0.07, r^{2}=0.00311$ and $P=0.06$, $r^{2}=0.40517$, respectively, at 3 months and 6 months after surgery; rim volume $P=0.06, r^{2}=0.00147$ and $P=0.06$, $r^{2}=0.26711$, respectively). Any significant changes also in cup shape measure were observed.

Changes in functional parameters ( $\Delta$ MD and $\Delta$ PSD) did not show statistically significant correlation with changes in rim indices ( $\Delta$ RIM area and $\Delta$ RIM volume) in both follow-up periods (Figures $2 \mathrm{a}$ and $\mathrm{b}$ and $3 \mathrm{a}$ and $b$ ).

\section{Discussion}

This study aimed at investigating the short-term morphological and functional effects of surgical IOP reduction in glaucoma. Using HRT and SAP, we showed that statistically significant changes in RNFL occurred 3 and 6 months after glaucoma filtering surgery, whereas

Table 2 Intraocular pressure, visual field indexes and optic nerve head parameters before and after glaucoma filtration surgery

\begin{tabular}{lccc}
\hline & Pre-operative & 3-months follow-up & 6-months follow-up \\
\hline IOP (mm Hg) & $24.4 \pm 5.00$ & $12.1 \pm 3.1 ; P<0.001$ & $10.6 \pm 2.8 ; P<0.001$ \\
MD SAP (dB) & $-14.05 \pm 3.37$ & $-13.35 \pm 3.26 ; P=0.06$ & $-13.58 \pm 3.54 ; P=0.06$ \\
PSD SAP & $8.58 \pm 1.79$ & $9.20 \pm 1.86 ; P=0.08$ & $8.97 \pm 1.52 ; P=0.06$ \\
Mean RNFL (mm) & $0.19 \pm 0.034$ & $0.24 \pm 0.039 ; P=0.05$ & $0.21 \pm 0.037 ; P=0.05$ \\
RNFL change (mm)/1-mm HG & & $0.009 \pm 0.004$ (range $0.005-0.013)$ & $0.004 \pm 0.002$ (range $0.002-0.006)$ \\
of IOP reduction & & & \\
Rim area $\left(\mathrm{mm}^{2}\right)$ & $1.15 \pm 0.59$ & $1.38 \pm 0.56 ; P=0.07$ & $1.27 \pm 0.42 ; P=0.06$ \\
Rim volume $\left(\mathrm{mm}^{3}\right)$ & $0.22 \pm 0.16$ & $0.27 \pm 0.18 ; P=0.06$ & $0.25 \pm 0.14 ; P=0.06$ \\
Cup area $\left(\mathrm{mm}^{2}\right)$ & $1.21 \pm 0.62$ & $0.96 \pm 0.47 ; P=0.06$ & $0.93 \pm 0.45 ; P=0.06$ \\
Cup volume $\left(\mathrm{mm}^{3}\right)$ & $0.36 \pm 0.19$ & $0.27 \pm 0.17 ; P=0.07$ & $0.24 \pm 0.16 ; P=0.06$ \\
Cup/disc area ratio & $0.52 \pm 0.25$ & $0.47 \pm 0.22 ; P=0.13$ & $0.48 \pm 0.21 ; P=0.13$ \\
Maximum cup depth $(\mathrm{mm})$ & $0.70 \pm 0.23$ & $0.65 \pm 0.25 ; P=0.18$ & $0.59 \pm 0.26 ; P=0.19$ \\
Cup shape measure & $-0.114 \pm 0.09$ & $-0.124 \pm 0.10 ; P=0.08$ & $-0.120 \pm 0.10 ; P=0.09$ \\
\hline
\end{tabular}

Values are expressed as mean \pm standard deviation. 

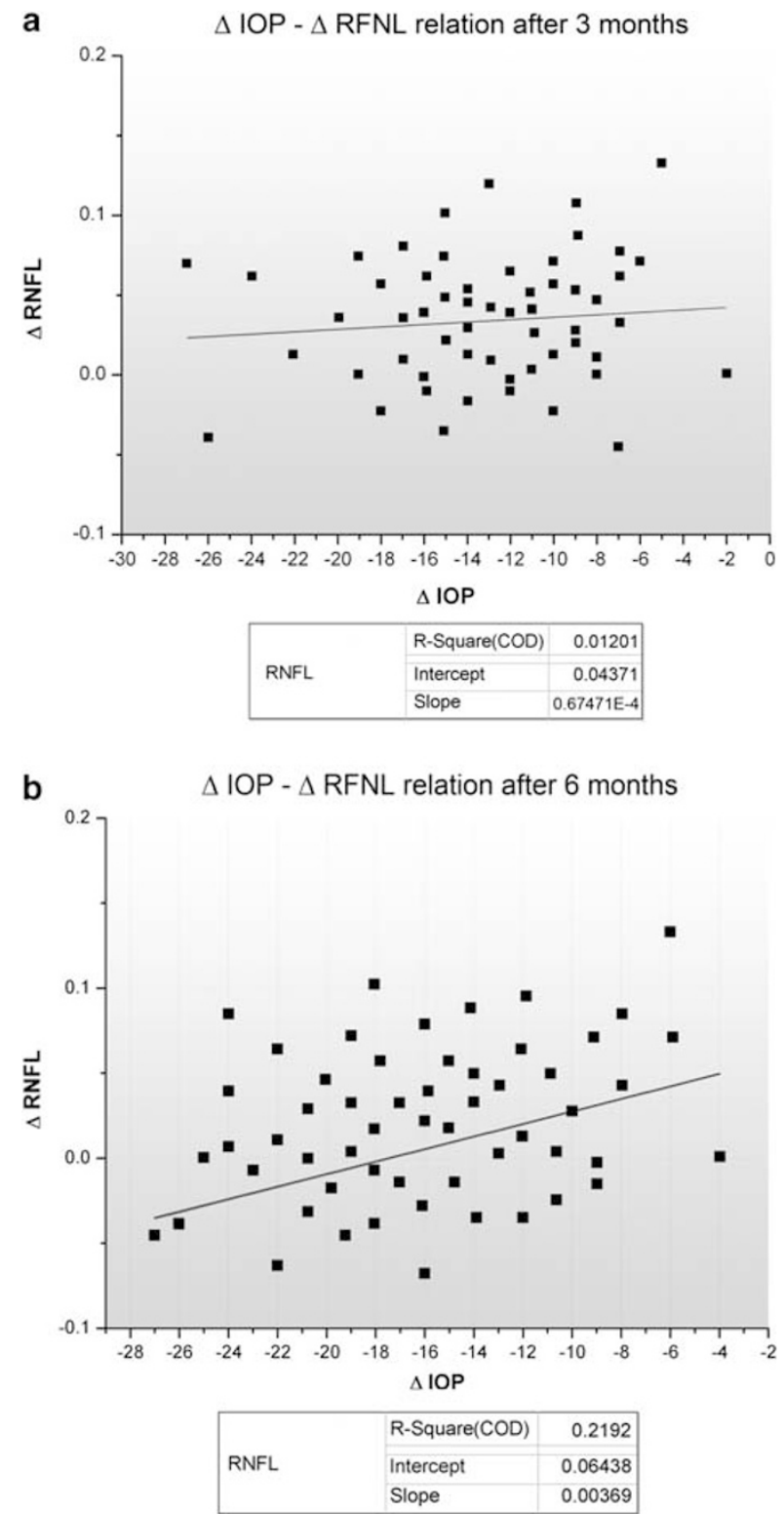

Figure 1 Relation $\Delta$ intraocular pressure $-\Delta$ retinal nerve fiber layer 3 (a) and 6 months (b) after surgery.

borderline $\mathrm{ONH}$ changes and negligible functional changes were found.

The lack of an absolute structure-function correlation may be due to the different levels of measurement noise displayed by the devices used to detect change, and it has been suggested that structural and functional methods can be considered as independent indicators of glaucoma damage. ${ }^{8,17,18}$ Scanty literature is available on the association of functional and morphological changes after surgical IOP decrease. Only recently a paper with long-term data has been published, showing that one third of eyes continued to have progression of glaucoma a

a $\quad \Delta M D-\Delta \mathrm{RIM}$ relation after 3 months
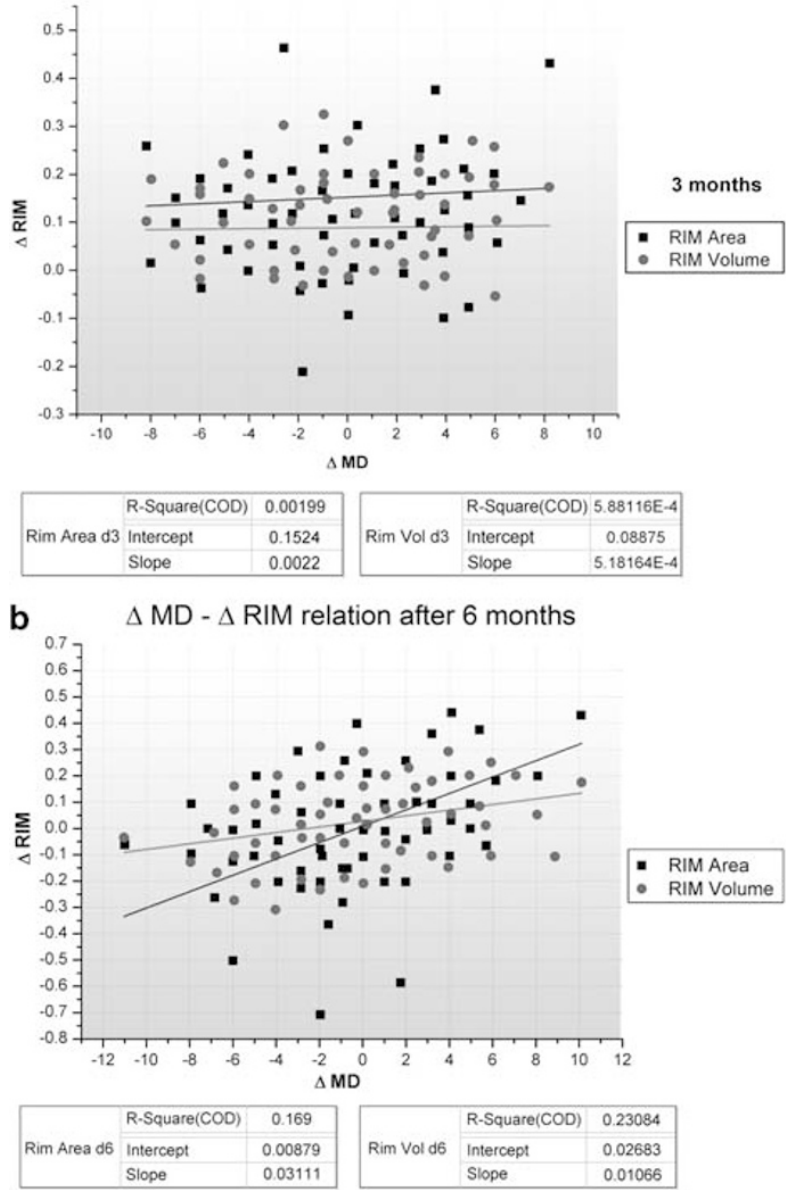

Figure 2 Relation $\Delta$ mean deviation $-\Delta$ rim parameters (area and volume) 3 (a) and 6 months (b) after surgery.

at 5 years after trabeculectomy. ${ }^{8}$ The authors suggest that the degree of IOP reduction after trabeculectomy may have an important role in the progression of glaucoma. Other authors found a small change of few microns of RNFL thickness ${ }^{4}$ or in $\mathrm{MD}^{5}$ after medical or surgical IOP reduction, even if the efficacy of the treatments was similar to our results.

Some authors described that a decrease of $\mathrm{ONH}$ cupping may occur due to an anterior movement of the lamina cribrosa. They postulated that when IOP was reduced after glaucoma filtering surgery, anterior repositioning of posteriorly displaced lamina cribrosa and simultaneous reformation of neuroretinal tissue would occur. ${ }^{19,20}$ Cup shape is a description of the distribution of depth values. Cups with gradually sloping borders tend to have more negative values, and cups with steep sloping walls tend to have less negative or even positive values. Thus, a change of the cup shape toward a more negative value represents an optic disk 
a
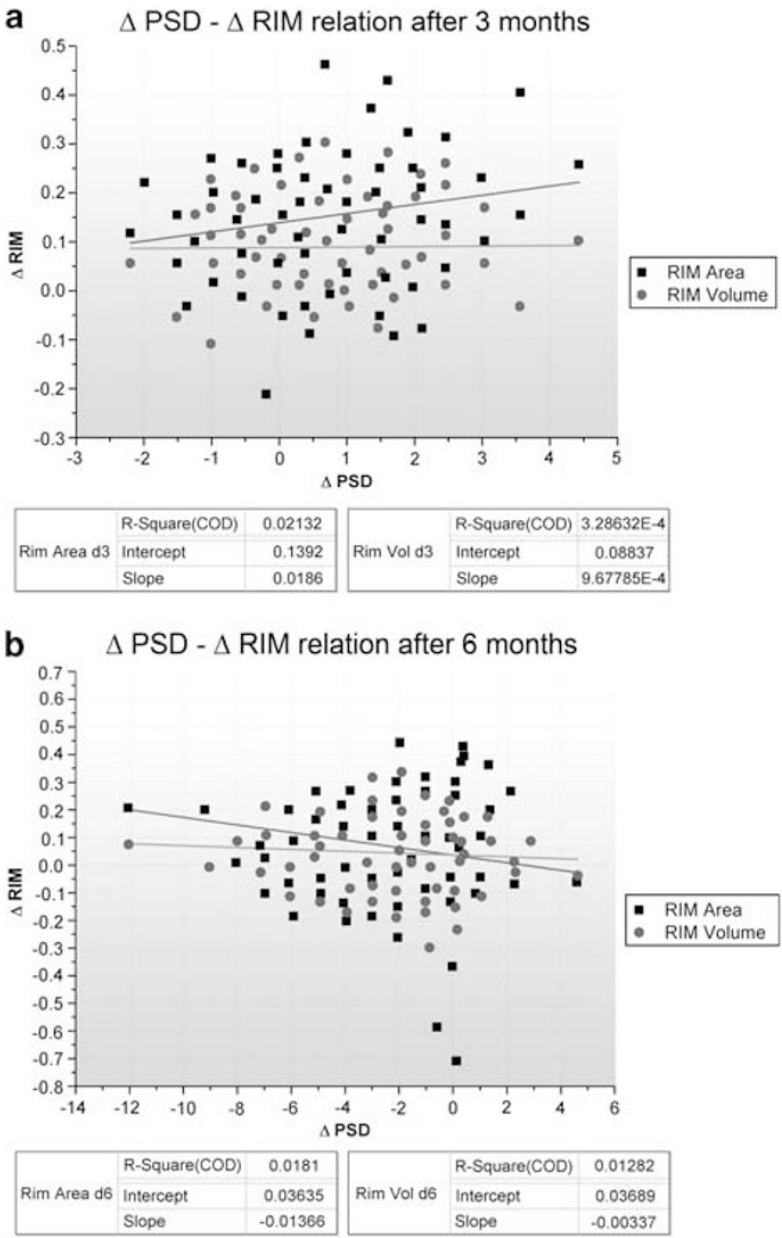

Figure 3 Relation $\Delta$ pattern standard deviation $-\Delta$ rim parameters (area and volume) 3 (a) and 6 months (b) after surgery.

improvement. In our series, the cup shape parameter became more negative than pre-operative cup shape, but the change was not significant. Another explanation could be $\mathrm{ONH}$ and peripapillary swelling due to the sudden post-operative IOP reduction. ${ }^{11}$ However, $\mathrm{ONH}$ changes are usually short-lived and one would not expect them to be present at 3 and 6 months.

On the basis of our results, we raise the hypothesis that the increase of RNFL thickness may reflect the recovery of the compressed RNFL, which would regain its original shape thanks to IOP reduction. As compression on the axons is relieved by IOP reduction, the axons may recover their normal shape and size, with resultant increase in RNFL thickness without changes in $\mathrm{ONH}$ parameters. These findings could be explained by the fact that the RNFL is a more direct measure of the integrity of retinal ganglion cells, in contrast to the neuroretinal rim, which is composed of nerve fiber bundles, glial cells, and connective tissues ${ }^{21}$ and could explain the presence of a statistically significant change for RNFL but not for ONH in our series. However, data interpretation deserves high caution because they could be also explained by physics rules. In fact, hydrostatic pressure and the normal osmotic pressure are interchangeable. Given a certain amount of colloids in the cytoplasm, it would be natural for cells to become larger when confined in a chamber of lower pressure than before, and may not say anything about the impending malfunction or death of the axon and ganglion cell.

Despite relevant inter-patients variations (Table 2), a change of $0.9 \mu \mathrm{m}$ and $0.4 \mu \mathrm{m}$ of thickening of the mean RNFL for each 1-mm Hg decrease of the IOP would occur at 3 and 6 months after filtration surgery. These findings are comparable with other studies in which the changes in RNFL height were shown by linear regression model to be pressure dependent. ${ }^{6,10}$ In particular, segmental analysis showed a significant change in rim volume in the nasal, inferonasal, superonasal, and superotemporal sectors. ${ }^{7}$ These authors have suggested that the improvement is limited to the nasal hemidisc and the adjacent RNFL because this area tends to be least affected by the disease, so nerve fiber bundle compression is not as long-standing as that within the temporal hemidisc. In our study, we analyzed only global $\mathrm{ONH}$ data and there may have been regional differences in optic disk topography that were ignored. Furthermore, our results may have been influenced by the fact that the sensitivity of HRT global indices to detect subtle ONH changes is probably minor than sectorial analysis. Additionally, even if we showed that RNFL change was associated with IOP change, the most relevant finding was the marked spread of data, highlighting a very high inter-patients variability as shown by scattergrams in Figures $1 \mathrm{a}$ and $\mathrm{b}$.

Another potential limiting factor of our study could be represented by the wide field of HRT images. Although smaller fields may enable the detection of subtle changes, it should be noted that other authors were able to detect statistically significant changes with $15^{\circ}$-field images. ${ }^{6}$

Our results confirm a previous finding by our group, which on different settings showed no significant changes in short-term perimetric variability after trabeculectomy. ${ }^{22}$ Still, the use of a small number of VF tests per patient may limit our results, as larger series of tests are usually necessary to discriminate true change from fluctuation. Very recently, we also showed that long-term fluctuation depends on the GSS stage. ${ }^{23}$ In order to protect our study from this potential problem, we chose a homogeneous sample of patients having $\mathrm{GSS}=4$. On the other hand, this could be a potential limiting factor because the relationship between morphological and functional changes induced by trabeculectomy might have different patterns at varying stages of the disease. Probably, these findings may not be 
applicable to patients with less or more advanced disease. It would be also interesting to evaluate if a sudden IOP reduction by medical treatment may obtain similar findings.

It might also be possible that different results would have been obtained if other perimetries, which are supposed to be more sensitive to subtle changes in subtypes of ganglion cells, ${ }^{24}$ had been used.

In this study, we considered RNFL change as the primary outcome because this parameter has the strongest structure-function association and high sensitivity for glaucoma detection; therefore, it may provide a better surrogate measure to monitor glaucoma progression and recovery after a marked IOP reduction compared with the neuroretinal rim. Besides, RNFL attained a higher value in the change of visual sensitivity with respect to different levels of structural damage compared with neuroretinal rim measures. ${ }^{21}$

The availability of the elements for a correct sample size calculation was limited by the lack of homogenous data on the effect of sudden IOP reduction on RNFL. We assumed that a change of mean RNFL thickness, as occurring between middle and advanced glaucoma, could be clinically significant. ${ }^{15}$ Some works have reported lower standard deviations for RNFL thickness, ${ }^{25,26}$ but we decided to use the largest that had been reported. ${ }^{15}$ Also, in order to prevent the possibility of negative results due to approximate sample size calculation, we set to $5 \%$ both type I and II statistical errors. With this premises, the main outcome of the study was statistically significant and comparable to other reports. ${ }^{14,25}$ As the study might be potentially overpowered, we considered as negative the borderline $P$-values for the secondary outcomes. As a consequence, caution in data interpretation and verification of our results by other studies seems advisable.

Finally, because of the short follow-up period and contrasting literature, studies with longer follow-up could be more useful to investigate the long-term effects of trabeculectomy on $\mathrm{ONH}$ and $\mathrm{VF}^{8}$

Our results showed a short-term improvement in global RNFL thickness after trabeculectomy. However, no definitive functional conclusions can be reached from these anatomical changes upon lowering IOP. In fact, even if changes were small and, at least in some cases, probably negligible from a clinical perspective, the direction of morphological changes was quite univocal for both RNFL and ONH. In several cases, borderline $P$-values of 0.06 were obtained, but these changes may be worthy of note, as the cut-off of $P$-value of 0.05 is arbitrary. In conclusion, some corresponding changes are suggestive, small enough that they could not be secured by the usual statistical criterion, but close enough that they can't be ignored and declared absent either.

\section{Summary}

\section{What was known before}

- Contrasting data are reported about optic nerve head, retinal nerve fiber layer and visual function changes in short-term follow-up after trabeculectomy.

\section{What this study adds}

- A significant increase in retinal nerve fiber layer thickness occurs after intraocular pressure decrease but we were not able to demonstrate significant changes in optic nerve head parameters and visual field examinations; however this could not indicate that a change did not occur.

\section{Conflict of Interest}

The authors declare no conflict of interest.

\section{References}

1 Advanced Glaucoma Intervention Study. 2. Visual field test scoring and reliability. [No authors listed] Ophthalmology 1994; 101: 1445-1455.

2 Quigley HA. Childhood glaucoma. Results with trabeculectomy and study of reversible cupping. Ophthalmology 1982; 89: 219-226.

3 Schwart B, Kamamoto T, Nagin P. Measurement of reversibility of optic disc cupping and pallor in ocular hypertension and glaucoma. Ophthalmology 1985; 92: 1396-1407.

4 Chang PT, Sekhon N, Budenz DL, Feuer WJ, Park PW, Anderson DR. Effect of lowering intraocular pressure on optical coherence tomography measurement of peripapillary retinal nerve fiber layer thickness. Ophthalmology 2007; 114: 2252-2258.

5 Rebolleda G, Muñoz-Negrete FJ, Noval S. Evaluation of changes in peripapillary nerve fiber layer thickness after deep sclerectomy with optical coherence tomography. Ophthalmology 2007; 114: 488-493.

6 Irak I, Zangwill L, Garden V, Shakiba S, Weinreb RN. Change in optic disk topography after trabeculectomy. Am J Ophthalmol 1996; 122: 690-695.

7 Kotecha A, Siriwardena D, Fitzke FW, Hitchings RA, Khaw PT. Optic disc changes following trabeculectomy: longitudinal and localization of change. $\mathrm{Br} J$ Ophthalmol 2001; 85: 956-961.

8 Kotecha A, Spratt A, Bence C, Garway-Heath DF, Khaw PT, Viswanathan A. Optic disc and visual field changes after trabeculectomy. Invest Ophthalmol Vis Sci 2009; 50: 4693-4699.

9 Katz LJ, Spaeth GL, Cantor LB, Poryzees EM, Steinmann WC. Reversible optic disc cupping and visual field improvement in adults with glaucoma. Am J Ophthalmol 1989; 107: 485-492.

10 Lesk MR, Spaeth GL, Azuara-Blanco A, Araujo SV, Katz LJ, Terebuh AK et al. Reversal of optic disc cupping after glaucoma surgery analyzed with a scanning laser tomograph. Ophthalmology 1999; 106: 1013-1018.

11 Aydin A, Wollstein G, Lyn rice L, Fujimoto JG, Schuman JS. Optical coherence tomography assessment of retinal nerve fiber layer thickness changes after glaucoma surgery. Ophthalmology 2003; 110: 1506-1511. 
12 Brusini P, Filacorda S. Enhanced glaucoma staging system (GSS2) for classifying functional damage in glaucoma. J Glaucoma 2006; 15: 40-46.

13 Kamal DS, Viswanathan AC, Garway-Heath DF, Hitchings RA, Poinoosawmy D, Bunce C. Detection of optic disc change with the Heidelberg retina tomograph before confirmed visual field change in ocular hypertensives converting to early glaucoma. Br J Ophthalmol 1999; 83: 290-294.

14 Hosking SL, Flanagan JG. Prospective study design for the Heidelberg retina tomograph; the effect of change in focus setting. Graefes Arch Clin Exp Ophthalmol 1996; 234: 306-310.

15 Yüksel N, Altintas O, Ozkan B, Karadag S, Caglar Y. Discriminating ability of optical coherence tomography data in staging glaucomatous damage. Can J Ophthalmol 2009; 44: 297-307.

16 Vernon SA, Hawker MJ, Ainsworth G, Hillman JG, MacNab HK, Dua HS. Laser scanning tomography of the optic nerve head in a normal elderly population: the Bridlington Eye Assessment Project. Invest Ophthalmol Vis Sci 2005; 46: 2823-2828.

17 Strouthidis NG, Scott A, Peter NM, Garway-Heath DF. Optic disc and visual field progression in ocular hypertensive subjects: detection rates, specificity, and agreement. Invest Ophthalmol Vis Sci 2006; 47: 2904-2910.

18 Artes PH, Chauhan BC. Longitudinal changes in the visual field and optic disc in glaucoma. Prog Retin Eye Res 2005; 24 333-354.

19 Levy NS, Crapss EE. Displacement of optic nerve head in response to short-term intraocular pressure elevation in human eyes. Arch Ophthalmol 1984; 102: 782-786.
20 Yan DB, Coloma FM, Metheetrairut A, Trope GE Heathcote JG, Ethier CR. Deformation of the laminar cribrosa by elevated intraocular pressure. $\mathrm{Br} J$ Ophthalmol 1984; 78: 643-648.

21 Leung CK, Medeiros FA, Zangwill LM, Sample PA, Bowd C, Ng D et al. American Chinese glaucoma imaging study: a comparison of the optic disc and retinal nerve fiber layer in detecting glaucomatous damage. Invest Ophthalmol Vis Sci 2007; 48: 2644-2652.

22 Fogagnolo P, McNaught A, Centofanti M, Rossetti L, Orzalesi N. The effects of intraocular pressure reduction on perimetric variability in glaucomatous eyes. Invest Ophthalmol Vis Sci 2007; 48: 4557-4563.

23 Fogagnolo P, Sangermani C, Oddone F, Frezzotti P, Iester M, Figus $\mathrm{M}$ et al. Long term perimetric variability in patients with different stages of glaucoma. Br J Ophthalmol 2011; 95: 189-193.

24 Fogagnolo P, Rossetti L, Ranno S, Ferreras A, Orzalesi N. Short-wavelength automated perimetry and frequencydoubling technology perimetry in glaucoma. Prog Brain Res 2008; 173: 101-124.

25 Li S, Wang X, Li S, Wu G, Wang N. Evaluation of optic nerve head and retinal nerve fiber layer in early and advance glaucoma using frequency-domain optical coherence tomography. Graefes Arch Clin Exp Ophthalmol 2010; 248: 429-434.

26 Gonzalez-Hernandez M, Pablo LE, Armas-Dominguez K, de la Vega RR, Ferreras A, de la Rosa MG. Structurefunction relationship depends on glaucoma severity. $\mathrm{Br} J$ Ophthalmol 2009; 93: 1195-1199. 